\title{
POLYNOMIAL FOLDINGS AND RANK OF TENSORS
}

\author{
STEVEN P. DIAZ AND ADAM LUTOBORSKI
}

\begin{abstract}
We review facts about rank, multilinear rank, multiplex rank and generic rank of tensors as well as folding of a tensor into a matrix of multihomogeneous polynomials. We define the new concept of folding rank of tensors and compare its properties to other ranks. We review the concept of determinantal schemes associated to a tensor. Then we define the new concept of a folding generic tensor meaning that all its determinantal schemes behave generically. Our main theorem states that for "small" 3tensors, any folding generic tensor has generic rank, and the reverse does not always hold.
\end{abstract}

1. Introduction. The main tool of multilinear algebra is the concept of a tensor product of vector spaces whose elements are called tensors. However, most important applications of this formalism are not in algebra but in differential geometry, mechanics, quantum mechanics and more recently in numerical analysis $[\mathbf{1 7}, \mathbf{1 8}]$. One very important numerical invariant of a tensor is its rank, the smallest number of simple tensors needed to express the tensor. As the rank of a given tensor is NP-hard to compute other related invariants have been defined such as multilinear rank and multiplex rank. After reviewing material on these ranks we study polynomial foldings of tensors, a way of representing a tensor as a matrix of multihomogeneous multilinear polynomials. These have been introduced before, but we use them in new ways. They allow us to define the folding rank of a tensor which is related to multilinear and multiplex rank but gives new, different information about the tensor. We also use these polynomial foldings to define determinantal schemes associated to a tensor. Again, this has been done before, but we carry it further and get new information.

An important property of rank is that, given a fixed tensor product of vector spaces over the complex numbers there is a generic or typical

2010 AMS Mathematics subject classification. Primary 13C40, 14M12, 15A69.

Keywords and phrases. Multilinear algebra, tensor rank, determinantal schemes.

Received by the editors on November 1, 2013, and in revised form on October 31, 2014 .

DOI: $10.1216 / \mathrm{JCA}-2016-8-2-173$

Copyright (C)2016 Rocky Mountain Mathematics Consortium 
rank for that tensor product. "Most" tensors from that tensor product have that generic rank. However, given a specific tensor, it can be difficult to determine whether that tensor has generic rank. Using the determinantal schemes associated to a tensor we define what it means for a tensor to be folding generic, meaning that all its determinantal schemes behave in a generic way. The main theorem of this paper states that, for certain families of small tensors, any folding generic tensor must have generic rank, but the reverse is not true. One wonders whether this is true more generally than for only these small tensors and what other information about a tensor the determinantal schemes may contain.

In Section 2, we give background on rank including definitions and some properties of rank, multilinear rank, multiplex rank and generic rank. Section 3 defines and gives some properties of polynomial foldings of tensors and folding rank. Section 4 defines determinantal schemes associated to a tensor and folding generic, then proves the main theorem about small tensors. Finally, in Section 5, we apply all this to study the elasticity tensor important in mechanics.

Throughout the paper, we work over the complex numbers. Some constructions and results are valid for other fields, others require the complex numbers or at least some restriction such as algebraically closed. So, to be safe, we always assume the base field is the complex numbers.

2. Background on tensor rank. We start by giving some background on ranks of tensors that can be found in many places, for instance, Landsberg [18] or Kolda-Bader [16]. We define rank, multilinear rank, multiplex rank and generic rank and give some of their properties as well as examples to show how multilinear and multiplex rank relate to each other.

A tensor $T$ is an element of a tensor product of several vector spaces. In general, the number of vector spaces could be finite or infinite, and the dimensions of the vector spaces could be finite or infinite. However, for purposes of this paper, we will always assume that there are finitely many vector spaces, and they all are finite-dimensional. $T \in V_{1} \otimes \cdots \otimes V_{n}, \operatorname{dim} V_{i}=d_{i}<\infty$; such a $T$ is called an $n$th order tensor or simply $n$-tensor. Assume $V_{i}$ has basis $\left\{v_{i, j}\right\}, 1 \leq j \leq d_{i}$. 
Definition 2.1. $T$ is said to be a simple tensor if and only if $T=$ $a_{1} \otimes \cdots \otimes a_{n}$, for some $a_{i} \in V_{i}$. The rank of an arbitrary tensor $T$ is defined to be the smallest possible number of simple tensors in any expression of $T$ as a sum of simple tensors.

Let $U$ and $V$ be two finite-dimensional vector spaces. Recall the canonical isomorphism $\operatorname{Hom}(U, V) \cong U^{*} \otimes V$ given by $u^{*} \otimes v \in U^{*} \otimes V$, and $u \in U,\left(u^{*} \otimes v\right)(u)=u^{*}(u) v$. Combining this with the canonical isomorphism $U^{* *} \cong U$, we get $\operatorname{Hom}\left(U^{*}, V\right) \cong U \otimes V . T=u \otimes v \in U \otimes V$ is canonically identified with a linear transformation $T_{U}: U^{*} \rightarrow V$ defined by $T_{U}\left(u^{*}\right)=u^{*}(u) v$. Now let us apply this to $T \in V_{1} \otimes \cdots \otimes V_{n}$. For each $i, 1 \leq i \leq n, T$ can be canonically identified with a linear transformation $T_{i}: V_{i}^{*} \rightarrow V_{1} \otimes \cdots \otimes V_{i-1} \otimes V_{i+1} \otimes \cdots \otimes V_{n}$.

Definition 2.2. The multilinear rank of $T$ is defined to be the $n$-tuple of non-negative integers

$$
\operatorname{rank}_{1}(T)=\left(\operatorname{rank}\left(T_{1}\right), \ldots, \operatorname{rank}\left(T_{n}\right)\right)
$$

where by $\operatorname{rank}\left(T_{i}\right)$ we mean $T_{i}$ 's rank as a linear transformation.

A standard coordinate definition of multilinear rank can be found in [20]. The reason for the notation rank $_{1}$ will become clear when we define multiplex rank.

It is useful to also think of multilinear rank in terms of coordinates. Using our chosen bases $\left\{v_{i, j}\right\}$, we may write

$$
T=\sum_{i_{1}=1}^{d_{1}} \cdots \sum_{i_{n}=1}^{d_{n}} a_{i_{1}, \ldots, i_{n}} v_{1, i_{1}} \otimes \ldots \otimes v_{n, i_{n}}
$$

for some constants $a_{i_{1}, \ldots, i_{n}} \in \mathbb{C}$, so that $T$ can be represented as an $n$-dimensional array of complex numbers $T=\left[a_{i_{1}, \ldots, i_{n}}\right]$. To study $T_{i}$, think of $V_{1} \otimes \cdots \otimes V_{i-1} \otimes V_{i+1} \otimes \cdots \otimes V_{n}$ as the space of $(n-1)$ dimensional arrays of numbers $B=\left[b_{k_{1}, \ldots, k_{i-1}, k_{i+1}, \ldots, k_{n}}\right]$, again using our chosen bases. As a basis for $V_{i}^{*}$, choose the dual basis to the chosen 
basis for $V_{i},\left\{v_{i, 1}^{*}, \ldots, v_{i, d_{i}}^{*}\right\}$.

$$
T_{i}\left(v_{i, j}^{*}\right)=\sum_{k_{1}=1}^{d_{1}} \ldots \sum_{k_{i-1}=1}^{d_{i-1}} \sum_{k_{i+1}=1}^{d_{i+1}} \cdots \sum_{k_{n}=1}^{d_{n}}\left(a_{k_{1}, \ldots, k_{i-1}, j, k_{i+1}, \ldots, k_{n}} \bigotimes_{\substack{l=1 \\ l \neq i}}^{n} v_{l, k_{l}}\right)
$$

By fixing $j$ at each of $1,2, \ldots, d_{i}$ we can think of $T=\left[a_{k_{1}, \ldots, k_{n}}\right]$ as $d_{i}$ elements of $V_{1} \otimes \cdots \otimes V_{i-1} \otimes V_{i+1} \otimes \cdots \otimes V_{n}$, and $\operatorname{rank}\left(T_{i}\right)$ is the dimension of the span in $V_{1} \otimes \cdots \otimes V_{i-1} \otimes V_{i+1} \otimes \cdots \otimes V_{n}$ of these $d_{i}$ elements. We are taking $d_{i}$ slices of the $n$-dimensional array $\left[a_{k_{1}, \ldots, k_{n}}\right]$ to obtain $d_{i}(n-1)$-dimensional arrays and looking at their span. For a coordinate definition of slices see [16].

Proposition 2.3. Let $T \in V_{1} \otimes \cdots \otimes V_{n}, \operatorname{rank}(T)=r$ and $\operatorname{rank}_{1}(T)=$ $\left(r_{1}, \ldots, r_{n}\right)$. Then $r \geq r_{i}$ for all $i$.

This is well known and straightforward. In [18], Exercise 2.4.2 (4) is a special case. See our Proposition 2.6 for a more general statement with proof. For some results on which $n$-tuples are obtainable as multilinear ranks see [5].

There is an obvious generalization of multilinear rank: multiplex rank. Both were introduced in 1927 by Hitchcock [14], see also [15, 16]. Their treatment is based on coordinates. Ours is more abstract.

Multilinear rank looks at moving one $V_{i}$ to the other side, that is, $T \in V_{1} \otimes \cdots \otimes V_{n}$ becomes $T_{i}: V_{i}^{*} \rightarrow V_{1} \otimes \cdots \otimes V_{i-1} \otimes V_{i+1} \otimes \cdots \otimes V_{n}$. One could move two or more of the $V_{i}$ 's to the other side. For any partition of the set $\{1, \ldots, n\}$ as a disjoint union of two sets $\{1, \ldots, n\}=A \cup A^{\prime}$ a tensor $T \in V_{1} \otimes \cdots \otimes V_{n}$ can be canonically identified with a linear transformation

$$
T_{A}: \bigotimes_{a \in A} V_{a}^{*} \rightarrow \bigotimes_{a^{\prime} \in A^{\prime}} V_{a^{\prime}}
$$

$T_{A}=T_{A^{\prime}}^{*}$, so if we are only concerned about the ranks of these maps, we may assume $|A| \leq\left|A^{\prime}\right|$. Fix an integer $m, 1 \leq m \leq n / 2$. Let $A_{i}$, $1 \leq i \leq\left(\begin{array}{c}n \\ m\end{array}\right)$ be the subsets of $\{1, \ldots, n\}$ of size $m$ in lexicographic order, and let $A_{i}^{\prime}$ be the complement of $A_{i}$ in $\{1, \ldots, n\}$. 
Definition 2.4. The size $m$ multiplex rank of $T$ is the $\left(\begin{array}{c}n \\ m\end{array}\right)$-tuple of non-negative integers

$$
\operatorname{rank}_{m}(T)=\left(\operatorname{rank}\left(T_{A_{1}}\right), \ldots, \operatorname{rank}\left(T_{A_{\left(\begin{array}{c}
n \\
m
\end{array}\right)}}\right)\right) .
$$

When $m=1$, we obtain the multilinear rank. This explains the notation for multilinear rank. As with multilinear rank, we can think of size $m$ multiplex rank in terms of taking slices of the $n$ dimensional array $\left[a_{i_{1}, \ldots, i_{n}}\right]$. In multilinear rank, we took one vector space to the other side, so we obtained a one-dimensional family of $(n-1)$-dimensional slices. In size $m$ multiplex rank we took $m$ vector spaces to the other side so we obtained an $m$-dimensional family of $(n-m)$-dimensional slices. It is easy to find examples which show that certain multiplex ranks sometimes distinguish between tensors that other multiplex ranks do not.

Example 2.5. Consider four vector spaces $U, V, W$ and $X$ with bases $\left\{u_{1}, u_{2}\right\},\left\{v_{1}, v_{2}\right\},\left\{w_{1}, w_{2}\right\}$ and $\left\{x_{1}, x_{2}\right\}$. Consider the following three tensors in $U \otimes V \otimes W \otimes X$.

$$
\begin{aligned}
& P=u_{1} \otimes v_{1} \otimes w_{1} \otimes x_{1}+u_{2} \otimes v_{2} \otimes w_{2} \otimes x_{2}, \\
& Q=u_{1} \otimes v_{1} \otimes w_{1} \otimes x_{1}+u_{2} \otimes v_{2} \otimes w_{2} \otimes x_{2}+u_{1} \otimes v_{2} \otimes w_{1} \otimes x_{2}, \\
& R=u_{1} \otimes v_{1} \otimes w_{1} \otimes x_{1}+u_{1} \otimes v_{2} \otimes w_{2} \otimes x_{2} .
\end{aligned}
$$

Compute the following:

$$
\begin{array}{ll}
\operatorname{rank}_{1}(P)=(2,2,2,2) & \operatorname{rank}_{2}(P)=(2,2,2,2,2,2) \\
\operatorname{rank}_{1}(Q)=(2,2,2,2) & \operatorname{rank}_{2}(Q)=(3,2,3,3,2,3) \\
\operatorname{rank}_{1}(R)=(1,2,2,2) & \operatorname{rank}_{2}(R)=(2,2,2,2,2,2) .
\end{array}
$$

$P$ and $Q$ have the same multilinear rank and different size 2 multiplex ranks. $P$ and $R$ have the same size 2 multiplex rank and different multilinear ranks. Neither rank determines the other.

As with multilinear rank, multiplex rank also provides lower bounds for ranks.

Proposition 2.6. Let $\operatorname{rank}(T)=r$. Let $g$ be any component of any multiplex rank of $T$. Then $g \leq r$. 
Proof. First, it is easy to see that if $\operatorname{rank}(T)=1$, that is, if $T$ is a simple tensor, then $\operatorname{rank} T_{A_{i}}=1$. If $\operatorname{rank}(T)=r$, then $T$ is a sum of $r$ simple tensors so $T_{A_{i}}$ is a sum of $r$ linear transformations of rank 1 and thus has rank at most $r$.

Notice that, in Example 2.5, size 2 multiplex rank gives a larger lower bound for rank than multilinear rank.

It follows from the description of rank in [18, subsection 5.2] that, for each $r$, the set $R_{r}=\left\{T \in V_{1} \otimes \cdots \otimes V_{n}: \operatorname{rank}(T)=r\right\}$ is constructible in the Zariski topology, see [13] for constructible and Zariski topology. Thus, there exists a unique $r$ such that $R_{r}$ contains a dense Zariski open subset. This uses that the complex numbers are algebraically closed. Over the real numbers this conclusion is not valid, but a related statement can be proven, [18, subsection 5.2.3].

Definition 2.7. This $r$ is called the generic rank of a tensor $T \in$ $V_{1} \otimes \cdots \otimes V_{n}$.

"Most" $T \in V_{1} \otimes \cdots \otimes V_{n}$ have generic rank.

3. Polynomial foldings of tensors. Previous authors (for instance, $[\mathbf{7}, \mathbf{1 1}]$ ) have shown how, starting from a tensor, one may in a natural manner construct various morphisms of free modules over polynomial rings. With respect to appropriate bases for these free modules the morphisms are represented by matrices with polynomial entries. We call these morphisms of free modules and their representing matrices polynomial foldings of the tensor. We define the folding rank of a tensor to be an ordered tuple listing the ranks of these matrices. We derive some properties of the folding rank and show how it relates to multilinear rank and size 2 multiplex rank.

We are not sure where the concept of folding of tensors originated. What we present here is based on material in $[\mathbf{7}, \mathbf{1 1}]$. First off, recall that, for any vector space $V$ over a field $F$, the symmetric algebra on $V$

$$
\mathrm{S}(V)=\bigoplus_{i=0}^{\infty} \mathrm{S}^{i}(V)
$$


is isomorphic to a polynomial ring over $F$. One may take as the set of variables any basis for $V$. Following the notation of [11], we denote this polynomial ring by $F[V]$. Now let $T \in V_{1} \otimes \cdots \otimes V_{n}$ be a tensor. As in Section 2, we may canonically identify $T$ with a linear transformation $T_{i}: V_{i}^{*} \rightarrow V_{1} \otimes \cdots \otimes V_{i-1} \otimes V_{i+1} \otimes \cdots \otimes V_{n}$ for each $i=1, \ldots, n$. From this, for each $j \neq i, 1 \leq j \leq n$, we construct a map of free modules over a polynomial ring.

$$
\phi_{i, j}: V_{i}^{*} \otimes F\left[\bigoplus_{l \neq i, j} V_{l}\right] \longrightarrow V_{j} \otimes F\left[\bigoplus_{l \neq i, j} V_{l}\right](1, \ldots, 1) .
$$

To define this map, we give its action on an element of the form $v_{i}^{*} \otimes f$, $v_{i}^{*} \in V_{i}^{*}, f \in F\left[\bigoplus_{l \neq i, j} V_{l}\right] . T_{i}\left(v_{i}^{*}\right) \in V_{1} \otimes \cdots \otimes V_{i-1} \otimes V_{i+1} \otimes \cdots \otimes V_{n}$, that is, $T_{i}\left(v_{i}^{*}\right)=\sum_{s=1}^{S} v_{1}^{(s)} \otimes \cdots \otimes v_{i-1}^{(s)} \otimes v_{i+1}^{(s)} \otimes \cdots \otimes v_{n}^{(s)}$. Then

$$
\phi_{i, j}\left(v_{i}^{*} \otimes f\right)=\sum_{s=1}^{S} v_{j}^{(s)} \otimes\left(\prod_{k \neq i, j} v_{k}^{(s)}\right) f .
$$

The $(1, \ldots, 1)$ in $(3.1)$ indicates that $\phi_{i, j}$ is a multigraded homomorphism. $F\left[\bigoplus_{l \neq i, j} V_{l}\right]$ is a multigraded ring, the multidegree of a monomial being its degrees in each of the $n-2$ sets of variables corresponding to the bases for each of the $n-2$ vector spaces in the direct sum. The $(1, \ldots, 1)$ indicates that the map sends something of multidegree $\left(e_{1}, \ldots, e_{n}\right)$ (remember we skip $e_{i}$ and $e_{j}$ ) to something of multidegree $\left(e_{1}+1, \ldots, e_{n}+1\right)$. The $\phi_{i, j}$ are the polynomial foldings of $T$. Notice that there are $2\left(\begin{array}{l}n \\ 2\end{array}\right)$ of them. We call them polynomial foldings because, when one chooses appropriate bases for the two free modules, $\phi_{i, j}$ is represented by a matrix whose entries are multihomogeneous multilinear polynomials. We discuss such matrices next.

We now write down polynomial folding in terms of coordinates. Let $T \in V_{1} \otimes \cdots \otimes V_{n}$ be a tensor. As before, with respect to the chosen bases, one may represent $T$ as an $n$-dimensional array of numbers $\left[a_{i_{1}, \ldots, i_{n}}\right]$. To define a folding of $T$ we must choose which $n-2$ indices get folded and which 2 indices remain unfolded. Say that $r$ and $s$ are the two indices not to be folded, $1 \leq r<s \leq n$. The folding of $T$ will be an $d_{r} \times d_{s}$ matrix $M$. It will be the matrix that represents $\phi_{s, r}$ with respect to the following bases for its domain and target. Let $\left\{v_{s, j}^{*}\right\}$, $1 \leq j \leq d_{s}$ be the basis of $V_{s}^{*}$ dual to $\left\{v_{s, j}\right\}$. As the basis for the 
domain, take $\left\{v_{s, j}^{*} \otimes 1\right\}$. As the basis for the target, take $\left\{v_{r, j} \otimes 1\right\}$. Each entry of $M$ will be a polynomial multilinear in the $n-2$ sets of variables corresponding to the $n-2$ bases corresponding to the $n-2$ vector spaces in the $n-2$ indices being folded. Here is the formula for the $(p, q)$ entry of $M$.

$$
[M]_{p, q}=\sum_{i_{1}=1}^{d_{1}} \ldots \sum_{i_{r}=p}^{p} \cdots \sum_{i_{s}=q}^{q} \ldots \sum_{i_{n}=1}^{d_{n}} a_{i_{1}, \ldots, i_{n}} \prod_{j \in\{1, \ldots, n\} \backslash\{r, s\}} v_{j, i_{j}} .
$$

Note that one may retrieve the original tensor $T$ from the folded matrix $M$ and the choice of bases. The matrix for $\phi_{j, i}$ is the transpose of the matrix for $\phi_{i, j}$, so we often assume $i<j$ and consider only $\left(\begin{array}{l}n \\ 2\end{array}\right)$ polynomial foldings rather than $2\left(\begin{array}{l}n \\ 2\end{array}\right)$.

It might be helpful to write this down with slightly different notation in the case where $n=3$. Let $U, V$ and $W$ be vector spaces of dimensions $d_{1}, d_{2}$ and $d_{3}$ and bases $\left\{u_{i}\right\},\left\{v_{j}\right\}$ and $\left\{w_{k}\right\}$, respectively. A tensor $T \in U \otimes V \otimes W$ can be represented with respect to the given bases by a three-dimensional array of numbers $\left[a_{i, j, k}\right]$. There are three possible foldings. We denote the three corresponding matrices of homogeneous linear polynomials by $M^{1}, M^{2}$ and $M^{3}$ depending on whether we fold along $U, V$ or $W$, respectively. The $(p, q)$ entries of these matrices are as follows.

$$
\begin{aligned}
& M_{p, q}^{1}=\sum_{i=1}^{d_{1}} a_{i, p, q} u_{i} \\
& M_{p, q}^{2}=\sum_{j=1}^{d_{2}} a_{p, j, q} v_{j} \\
& M_{p, q}^{3}=\sum_{k=1}^{d_{3}} a_{p, q, k} w_{k} .
\end{aligned}
$$

Certain of these folding matrices are almost identical to certain pencil matrices used in classifying small tensors, [18, subsection 3.11, Chapter 10].

For a matrix $M$ with entries in a ring $R$ it is standard to define the rank of $M$ to be the largest integer $r$ such that $M$ has a nonzero 
$r \times r$ subdeterminant. Our folding matrices have polynomial entries, so the ring $R$ in this case is an integral domain. The rank then can also be computed by thinking of $M$ as a matrix with entries in a field-the fraction field of $R$. All the equivalent ways of computing the rank of a matrix with entries in a field may be used.

Let $T \in V_{1} \otimes \cdots \otimes V_{n}$ be a tensor. We saw that, for each pair of integers $1 \leq r<s \leq n$ we obtain from $T$ a folded matrix, which we shall denote by $M(r, s)$ or, if needed, $M_{T}(r, s)$. Order the pairs lexicographically $(1,2)<(1,3)<\cdots<(1, n)<(2,3)<\cdots<$ $(n-1, n)$. The number of such pairs is given by the binomial coefficient $\left(\begin{array}{l}n \\ 2\end{array}\right)$.

Definition 3.1. The folding rank of the tensor $T$ is the $\left(\begin{array}{l}n \\ 2\end{array}\right)$-tuple of integers

$$
\operatorname{rankf}(T)=(\operatorname{rank}(M(1,2)), \ldots, \operatorname{rank}(M(n-1, n)))
$$

ordered lexicographically.

We leave it to the reader to check that this is independent of the choice of bases.

Just as in the case of multilinear rank, rank is an upper bound for every component of folding rank.

Proposition 3.2. Let $T \in V_{1} \otimes \cdots \otimes V_{n}, \operatorname{rank}(T)=r$ and $\operatorname{rankf}(T)=$ $\left(r_{1}, \ldots, r_{\left(\begin{array}{c}n \\ 2\end{array}\right)}\right)$. Then $r \geq r_{i}$ for all $i$.

Proof. It is easy to see that for a simple tensor every component of the folding rank equals 1 . It is also easy to see that

$$
M_{T_{1}+T_{2}}(k, l)=M_{T_{1}}(k, l)+M_{T_{2}}(k, l),
$$

the folding of a sum of tensors equals the sum of the foldings of those tensors. Write $T$ as a sum of $r$ simple tensors. Then every folding of $T$ is a sum of $r$ matrices each of rank 1 and thus has rank at most $r$.

Example 3.3. When $n=3$, both the multilinear rank and the folding rank are ordered triples of integers. One may wonder whether they are equal or whether one determines the other. Here we give examples 
that show the answers to these questions are negative. These examples come from the proof of Theorem 4.6. Let $U$ have basis $\left\{u_{1}, u_{2}\right\}, V$ have basis $\left\{v_{1}, v_{2}, v_{3}\right\}$ and $W$ have basis $\left\{w_{1}, w_{2}, w_{3}\right\}$. Consider the following three tensors:

$$
\begin{gathered}
A=u_{1} \otimes v_{1} \otimes w_{1}+u_{1} \otimes v_{2} \otimes w_{2}+u_{2} \otimes v_{2} \otimes w_{1}+u_{2} \otimes v_{3} \otimes w_{2} \\
B=u_{1} \otimes v_{1} \otimes w_{1}+u_{1} \otimes v_{2} \otimes w_{3}+u_{2} \otimes v_{1} \otimes w_{2}+u_{2} \otimes v_{3} \otimes w_{3} \\
C=u_{1} \otimes v_{1} \otimes w_{1}+u_{1} \otimes v_{2} \otimes w_{2}+u_{2} \otimes v_{3} \otimes w_{3} \\
A_{1}\left(u_{1}^{*}\right)=\left[\begin{array}{lll}
1 & 0 & 0 \\
0 & 1 & 0 \\
0 & 0 & 0
\end{array}\right], \quad A_{1}\left(u_{2}^{*}\right)=\left[\begin{array}{lll}
0 & 0 & 0 \\
1 & 0 & 0 \\
0 & 1 & 0
\end{array}\right] \\
A_{2}\left(v_{1}^{*}\right)=\left[\begin{array}{lll}
1 & 0 & 0 \\
0 & 0 & 0
\end{array}\right], \quad A_{2}\left(v_{2}^{*}\right)=\left[\begin{array}{lll}
0 & 1 & 0 \\
1 & 0 & 0
\end{array}\right], \quad A_{2}\left(v_{3}^{*}\right)=\left[\begin{array}{lll}
0 & 0 & 0 \\
0 & 1 & 0
\end{array}\right] \\
A_{3}\left(w_{1}^{*}\right)=\left[\begin{array}{lll}
1 & 0 & 0 \\
0 & 1 & 0
\end{array}\right], \quad A_{3}\left(w_{2}^{*}\right)=\left[\begin{array}{lll}
0 & 1 & 0 \\
0 & 0 & 1
\end{array}\right], \quad A_{3}\left(w_{3}^{*}\right)=\left[\begin{array}{lll}
0 & 0 & 0 \\
0 & 0 & 0
\end{array}\right]
\end{gathered}
$$

We conclude that $\operatorname{rank}_{1}(A)=(2,3,2)$. For $A$, we have:

$$
M^{1}=\left[\begin{array}{ccc}
u_{1} & 0 & 0 \\
u_{2} & u_{1} & 0 \\
0 & u_{2} & 0
\end{array}\right], \quad M^{2}=\left[\begin{array}{ccc}
v_{1} & v_{2} & 0 \\
v_{2} & v_{3} & 0
\end{array}\right], \quad M^{3}=\left[\begin{array}{ccc}
w_{1} & w_{2} & 0 \\
0 & w_{1} & w_{2}
\end{array}\right] .
$$

We conclude that $\operatorname{rankf}(A)=(2,2,2)$. In a similar manner, one computes that $\operatorname{rank}_{1}(B)=(2,3,3)$ and $\operatorname{rankf}(B)=(2,2,2)$ and that $C$ has $\operatorname{rank}_{1}(C)=(2,3,3)$ and $\operatorname{rankf}(C)=(3,2,2) . A$ and $B$ have the same folding ranks but different multilinear ranks. $B$ and $C$ have the same multilinear ranks, but different folding ranks. Multilinear rank does not determine folding rank, and folding rank does not determine multilinear rank.

Notice that, in the example, $M^{1}=u_{1} A_{1}\left(u_{1}^{*}\right)+u_{2} A_{1}\left(u_{2}^{*}\right), M^{2}=$ $v_{1} A_{2}\left(v_{1}^{*}\right)+v_{2} A_{2}\left(v_{2}^{*}\right)+v_{3} A_{2}\left(v_{3}^{*}\right)$ and $M^{3}=w_{1} A_{3}\left(w_{1}^{*}\right)+w_{2} A_{3}\left(w_{2}^{*}\right)+$ $w_{3} A_{3}\left(w_{3}^{*}\right)$. For 3 -tensors, this always happens.

Proposition 3.4. Let $T \in U \otimes V \otimes W$. Then

$$
M^{1}=\sum_{i=1}^{d_{1}} u_{i} T_{1}\left(u_{i}^{*}\right), \quad M^{2}=\sum_{j=1}^{d_{2}} v_{j} T_{2}\left(v_{j}^{*}\right), \quad M^{3}=\sum_{k=1}^{d_{3}} w_{k} T_{3}\left(w_{k}^{*}\right) .
$$


Proof. Compare the explicit description of $M^{i}$ given in equation (3.3) with the description of multilinear rank in terms of slices given directly before Proposition 2.3.

Corollary 3.5. Let $T \in U \otimes V \otimes W$. By definition,

$$
\operatorname{rank}_{1}(T)=\left(\operatorname{dim}\left(T_{1}\left(U^{*}\right)\right), \operatorname{dim}\left(T_{2}\left(V^{*}\right)\right), \operatorname{dim}\left(T_{3}\left(W^{*}\right)\right)\right) .
$$

Set $r_{1}$ to be the largest rank of any matrix in $T_{1}\left(U^{*}\right), r_{2}$ the largest rank of any matrix in $T_{2}\left(V^{*}\right), r_{3}$ the largest rank of any matrix in $T_{3}\left(W^{*}\right)$. Then

$$
\operatorname{rankf}(T)=\left(r_{1}, r_{2}, r_{3}\right)
$$

Proof. Concentrate on the first component of the triple. The others are similar. $M^{1}$ is a matrix with variable entries. By the proposition, as those variable entries are filled with all possible constant entries, the set of matrices obtained is exactly $T_{1}\left(U^{*}\right)$. The rank of a matrix is the largest size of a nonzero subdeterminant of that matrix. A subdeterminant of a matrix with variable entries is a polynomial in those variables. A polynomial is not the zero polynomial if and only if it takes on a nonzero value for some choice of constant values for its variables. Thus, the largest size of a nonzero subdeterminant of the matrix with variable entries is equal to the largest size of a nonzero subdeterminant of any of the corresponding matrices of constant entries obtained by assigning constant values to the variables. This gives the result.

The generalization of this result to tensors of order 4 or higher is much less elegant. First off, one cannot compare folding rank with multilinear rank because the lengths of the tuples are not even the same. To solve that, one instead compares folding rank to size 2 multiplex rank as, in both cases, one gets tuples of length $\left(\begin{array}{l}n \\ 2\end{array}\right)$.

Let $T \in V_{1} \otimes \cdots \otimes V_{n}, n>3$. Consider the folding $\phi_{i, j}$ of $T$. Set $A=\{i, j\}, A^{\prime}=\{1, \ldots, n\} \backslash\{i, j\}$. The matrix for $\phi_{i, j}$ will have entries that are variables corresponding to the bases for $V_{l}, l \in A^{\prime}$. For each choice of specific constant values of these variables one gets an element of $V_{i} \otimes V_{j}$.

Now consider the size 2 multiplex rank. We would look at the rank of the map $T_{A}: V_{i}^{*} \otimes V_{j}^{*} \rightarrow \bigotimes_{l \in A^{\prime}} V_{l}$. Instead we look at its dual 
$T_{A^{\prime}}: \bigotimes_{l \in A^{\prime}} V_{l}^{*} ; \rightarrow V_{i} \otimes V_{j}$, which has the same rank. This induces a multilinear map $\widetilde{T}_{A^{\prime}}: \bigoplus_{l \in A^{\prime}} V_{l}^{*} ; \rightarrow V_{i} \otimes V_{j}$ sending $\bigoplus_{l \in A^{\prime}} v_{l}^{*}$ to $T_{A^{\prime}}\left(\bigotimes_{l \in A^{\prime}} v_{l}^{*}\right)$. The image of $\widetilde{T}_{A^{\prime}}$ is the set of all elements of $V_{i} \otimes V_{j}$ one obtains by choosing all possible specific constant values for the variables in the matrix for $\phi_{i, j}$. This image linearly spans the image of $T_{A^{\prime}}$ but will not, in general, equal the image. To get the $A$ term for size 2 multiplex rank one computes the dimension of the image of $T_{A^{\prime}}$. To get this term for the folding rank one does not compute the largest rank of a matrix in the image of $T_{A^{\prime}}$, one computes the largest rank of any matrix in the image of $\widetilde{T}_{A^{\prime}}$, a subvariety of the image of $T_{A^{\prime}}$ that linearly spans this image.

Example 3.6. Consider four vector spaces $U, V, W$ and $X$ with bases $\left\{u_{1}, u_{2}\right\},\left\{v_{1}, v_{2}\right\},\left\{w_{1}, w_{2}\right\}$ and $\left\{x_{1}, x_{2}\right\}$. Consider $T \in U \otimes V \otimes W \otimes X$, $T=u_{1} \otimes v_{1} \otimes w_{1} \otimes x_{1}+u_{1} \otimes v_{2} \otimes w_{1} \otimes x_{2}+u_{2} \otimes v_{1} \otimes w_{2} \otimes x_{1}+u_{2} \otimes v_{2} \otimes w_{2} \otimes x_{2}$. The matrix for $\phi_{1,2}$ is:

$$
M=\left[\begin{array}{ll}
u_{1} v_{1} & u_{1} v_{2} \\
u_{2} v_{1} & u_{2} v_{2}
\end{array}\right] .
$$

The image of $T_{\{3,4\}}$ is spanned by the matrices

$$
\left[\begin{array}{ll}
1 & 0 \\
0 & 0
\end{array}\right], \quad\left[\begin{array}{ll}
0 & 1 \\
0 & 0
\end{array}\right], \quad\left[\begin{array}{ll}
0 & 0 \\
1 & 0
\end{array}\right], \quad\left[\begin{array}{ll}
0 & 0 \\
0 & 1
\end{array}\right] .
$$

The linear span of all matrices obtained by putting specific values into $M$ is all $2 \times 2$ matrices and thus contains matrices of $\operatorname{rank} 2$, but all matrices so obtained have rank at most 1 .

4. Determinantal schemes of foldings. This section contains the main theorem of this paper. When one has a matrix whose entries lie in a ring that is not a field, it is common practice in algebraic geometry and commutative algebra to study certain determinantal schemes associated to the matrix. See, for instance, [10] under determinantal rings and determinantal ideals and $[\mathbf{4}, \mathbf{8}, \mathbf{9}]$, as well as [13] for general information on schemes. The folded matrices obtained in Section 3 are this sort of matrix. We use this construction to define determinantal schemes associated to a tensor. In $[\mathbf{7}, \mathbf{1 1}]$, this has already been done to some extent. There is also a standard concept in algebraic geometry and commutative algebra of what it means for these determinantal 
schemes to behave generically, see $[\mathbf{2}, \mathbf{1 0}]$. We use this to define what it means for a tensor to be folding generic. The main theorem then shows that, for certain small tensors, any of these tensors that is folding generic is also of generic rank, but the reverse implication does not hold. We wonder whether this may also be true more generally than for only these small tensors. The material leading up to Definition 4.3 is standard. The material that follows is new.

Let $M$ be an $m \times n$ matrix with entries in a polynomial ring $F\left[x_{1}, \ldots, x_{t}\right], F$ a field. For each integer $1 \leq d \leq \min (m, n)$, define $I_{d}(M)$ to be the ideal in $F\left[x_{1}, \ldots, x_{t}\right]$ generated by all the $d \times d$ subdeterminants of $M$.

Definition 4.1. The $d$-th determinantal subscheme of $M$, denoted $D_{d}(M)$, is defined to be the subscheme of $F^{t}$ defined by the ideal $I_{d}(M)$.

As a set $D_{d}(M) \subset F^{t}$ is the common zeros of all the $d \times d$ subdeterminants of $M$, which is equal to the set $\left\{P=\left(a_{1}, \ldots, a_{t}\right) \in\right.$ $\left.F^{t}: \operatorname{rank}(M(P))<d\right\}$. By $M(P)$, we mean substituting $P=$ $\left(a_{1}, \ldots, a_{t}\right)$ into all the polynomial entries of $M$ to obtain a matrix with entries in $F$.

Definition 4.2. The scheme $D_{d}(M)$ is said to exhibit generic behavior if and only if it satisfies all the following conditions.

(i) $D_{d}(M)$ is a reduced scheme.

(ii) Every irreducible component of $D_{d}(M)$ has dimension equal to $t-(m-d+1)(n-d+1)$.

(iii) If $t-(m-d+1)(n-d+1)>0, D_{d}(M)$ is irreducible.

(iv) The singularities of $D_{d}(M)$, if any, are contained in $D_{d-1}(M)$.

It is well known $[\mathbf{2}, \mathbf{1 0}]$ that, if the entries of $M$ are filled generically, then for any $d$ the scheme $D_{d}(M)$ exhibits generic behavior.

Now, suppose all the entries of $M$ are homogeneous of the same degree or multihomogeneous of the same multidegree. We can then define $D_{d}(M)$ as a subscheme of the projective space $\mathbb{P}^{t-1}$, or an appropriate product of projective spaces. In the definition of generic behavior the only thing that changes is $t$ is replaced by $t-1$ or the dimension of the given product of projective spaces. 
Definition 4.3. Let $T \in V_{1} \otimes \cdots \otimes V_{n}$ be a tensor. We say that $T$ is folding generic if and only if, for every pair of integers $1 \leq r<s \leq n$, the folded matrix $M(r, s)$ has the property that all of its determinantal varieties exhibit generic behavior.

Remark 4.4. It is easy to see that, if $T$ is folding generic, then all components of its folding rank are as large as possible. That is, the component of the folding rank corresponding to $\phi_{i, j}$ will equal the minimum of $d_{i}$ and $d_{j}$. This is because the subdeterminants of the matrix for $\phi_{i, j}$ of largest possible size cannot all be identically 0 .

Remark 4.5. The referee suggested, "It might be useful to consider weaker genericity properties than those in Definition 4.2, to require, e.g., only that property 2 , or say properties 2 and 4 , hold, forgetting the others. This may give a notion that relates more closely to border rank." See [18] for border rank. We agree that this suggestion is worthy of investigation.

We now have two notions of generic behavior for a tensor: rank generic, meaning the tensor has generic rank, and folding generic. One may wonder how they relate to each other. We already saw in Section 2 that there exists a dense Zariski open subset $U_{1} \subset V_{1} \otimes \cdots \otimes V_{n}$ such that all tensors $T \in U_{1}$ are rank generic. Because choosing the entries of a matrix generically leads to generic behavior of determinantal varieties, it is likely that there exists a dense Zariski open subset $U_{2} \subset V_{1} \otimes \cdots \otimes V_{n}$ such that all tensors $T \in U_{2}$ are folding generic. So, the two notions are probably mostly the same. They are not, however, identical.

Theorem 4.6. Consider a tensor $T \in U \otimes V \otimes W$ where $\operatorname{dim} U=2$, $\operatorname{dim} V=2$ or 3 , and $\operatorname{dim} W \geq 2$. If $T$ is folding generic, then $T$ is rank generic. The reverse implication does not always hold.

Remark 4.7. Before giving the proof, we will explain the strategy of the proof and explain how it actually shows something slightly stronger than the theorem states. The product $G L(U) \times G L(V) \times G L(W)$ acts on $U \otimes V \otimes W$ by acting on each factor. This action is just a change of coordinates so whether a tensor is rank generic or folding generic is clearly invariant under this action. We may check the veracity of 
the theorem by checking it at one point in each orbit of the group action. From Landsberg [18, Chapter 10], we see that, under the dimension restrictions of the theorem, there are only finitely many orbits. Furthermore, [18] provides a list of all the orbits, from each orbit a specific element, and for most (enough for our purposes) orbits the dimension of the orbit. Thus, we can prove the theorem simply by checking that it is true on finitely many specific tensors. This is what we shall do.

It turns out that there is always exactly one orbit consisting of folding generic tensors. This is the generic orbit, the dense orbit, the orbit of largest dimension. By the remarks directly before the theorem, we knew that tensors from this orbit must be rank generic. We also find that sometimes there are other orbits consisting of rank generic tensors, but as previously indicated never any other orbits consisting of folding generic tensors.

Proof. We use the information in [18, Tables 10.3.1-10.3.5]. We follow its numbering of the orbits 1-26. $U$ will have basis $\left\{u_{1}, u_{2}\right\}, V$ will have basis $\left\{v_{1}, v_{2}\right\}$ or $\left\{v_{1}, v_{2}, v_{3}\right\}$, as appropriate, and $W$ will have basis $\left\{w_{1}, \ldots, w_{c}\right\}$. Thus, the three polynomial foldings of $T, M^{1}, M^{2}$ and $M^{3}$ will have entries that are homogeneous linear polynomials in $u_{i}$ 's, $v_{j}$ 's and $w_{k}$ 's, respectively. When [18] gives the dimension of an orbit, it is giving its dimension as a projective variety which is one less than its dimension as an affine variety. When we do not give the dimension of the orbit, it means that this information is not contained in $[\mathbf{1 8}]$.

Let us start with the cases where $V$ has dimension 2. These cases are covered in [18, Table 10.3.1].

Orbit 1 . This has dimension $c+1$ with typical element $u_{1} \otimes v_{1} \otimes w_{1}$ of rank $1 . M^{1}$ is $2 \times c, M^{2}$ is $2 \times c$ and $M^{3}$ is $2 \times 2$.

$$
\begin{aligned}
M^{1} & =\left[\begin{array}{ccc}
u_{1} & 0 & \ldots \\
0 & 0 & \ldots
\end{array}\right] \\
M^{2} & =\left[\begin{array}{lll}
v_{1} & 0 & \ldots \\
0 & 0 & \ldots
\end{array}\right] \\
M^{3} & =\left[\begin{array}{cc}
w_{1} & 0 \\
0 & 0
\end{array}\right] .
\end{aligned}
$$


None of these matrices exhibit generic behavior as their $(2 \times 2)$ minors are all identically 0 . The points in this orbit are not folding generic.

Orbit 2. This has dimension $c+2$ with typical element $u_{1} \otimes v_{1} \otimes$ $w_{1}+u_{2} \otimes v_{2} \otimes w_{1}$ of rank $2 . M^{1}$ is $2 \times c, M^{2}$ is $2 \times c$ and $M^{3}$ is $2 \times 2$.

$$
\begin{aligned}
M^{1} & =\left[\begin{array}{lll}
u_{1} & 0 & \ldots \\
u_{2} & 0 & \ldots
\end{array}\right] \\
M^{2} & =\left[\begin{array}{lll}
v_{1} & 0 & \ldots \\
v_{2} & 0 & \ldots
\end{array}\right] \\
M^{3} & =\left[\begin{array}{cc}
w_{1} & 0 \\
0 & w_{1}
\end{array}\right] .
\end{aligned}
$$

$M^{1}$ and $M^{2}$ do not exhibit generic behavior because their $(2 \times 2)$ minors are all identically $0 . M^{3}$ does not exhibit generic behavior because its determinant gives a non-reduced scheme. The points in this orbit are not folding generic.

Orbit 3. This has dimension $2 c$ with typical element $u_{1} \otimes v_{1} \otimes w_{1}+$ $u_{1} \otimes v_{2} \otimes w_{2}$ of rank 2. $M^{1}$ is $2 \times c, M^{2}$ is $2 \times c$ and $M^{3}$ is $2 \times 2$.

$$
\begin{aligned}
M^{1} & =\left[\begin{array}{cccc}
u_{1} & 0 & 0 & \ldots \\
0 & u_{1} & 0 & \ldots
\end{array}\right] \\
M^{2} & =\left[\begin{array}{cccc}
v_{1} & v_{2} & 0 & \ldots \\
0 & 0 & 0 & \ldots
\end{array}\right] \\
M^{3} & =\left[\begin{array}{cc}
w_{1} & w_{2} \\
0 & 0
\end{array}\right] .
\end{aligned}
$$

$M^{1}$ does not exhibit generic behavior because its $2 \times 2$ determinants give a non-reduced scheme. $M^{2}$ and $M^{3}$ do not exhibit generic behavior because their $2 \times 2$ determinants are all 0 . The points in this orbit are not folding generic.

Orbit 4 . This has dimension $2 c$ with typical element $u_{1} \otimes v_{1} \otimes w_{1}+$ 
$u_{2} \otimes v_{1} \otimes w_{2}$ of rank $2 . M^{1}$ is $2 \times c, M^{2}$ is $2 \times c$ and $M^{3}$ is $2 \times 2$.

$$
\begin{aligned}
M^{1} & =\left[\begin{array}{cccc}
u_{1} & u_{2} & 0 & \ldots \\
0 & 0 & 0 & \ldots
\end{array}\right] \\
M^{2} & =\left[\begin{array}{cccc}
v_{1} & 0 & 0 & \ldots \\
0 & v_{1} & 0 & \ldots
\end{array}\right] \\
M^{3} & =\left[\begin{array}{ll}
w_{1} & 0 \\
w_{2} & 0
\end{array}\right] .
\end{aligned}
$$

$M^{1}$ and $M^{3}$ do not exhibit generic behavior because their $2 \times 2$ determinants are all $0 . M^{2}$ does not exhibit generic behavior because its $2 \times 2$ determinants give a non-reduced scheme. The points in this orbit are not folding generic.

Orbit 5. This has dimension $2 c+2$ with typical element $u_{1} \otimes v_{1} \otimes$ $w_{1}+u_{1} \otimes v_{2} \otimes w_{2}+u_{2} \otimes v_{1} \otimes w_{2}$ of rank 3. $M^{1}$ is $2 \times c, M^{2}$ is $2 \times c$, and $M^{3}$ is $2 \times 2$.

$$
\begin{aligned}
M^{1} & =\left[\begin{array}{llll}
u_{1} & u_{2} & 0 & \ldots \\
0 & u_{1} & 0 & \ldots
\end{array}\right] \\
M^{2} & =\left[\begin{array}{llll}
v_{1} & v_{2} & 0 & \ldots \\
0 & v_{1} & 0 & \ldots
\end{array}\right] \\
M^{3} & =\left[\begin{array}{cc}
w_{1} & w_{2} \\
w_{2} & 0
\end{array}\right] .
\end{aligned}
$$

$M^{1}, M^{2}$ and $M^{3}$ do not exhibit generic behavior because their $2 \times 2$ determinants give non-reduced schemes. The points in this orbit are not folding generic.

Orbit 6. This has dimension $2 c+3$ with typical element $u_{1} \otimes v_{1} \otimes$ $w_{1}+u_{2} \otimes v_{2} \otimes w_{2}$ of rank $2 . M^{1}$ is $2 \times c, M^{2}$ is $2 \times c$ and $M^{3}$ is $2 \times 2$.

$$
\begin{aligned}
M^{1} & =\left[\begin{array}{cccc}
u_{1} & 0 & 0 & \ldots \\
0 & u_{2} & 0 & \ldots
\end{array}\right] \\
M^{2} & =\left[\begin{array}{cccc}
v_{1} & 0 & 0 & \ldots \\
0 & v_{2} & 0 & \ldots
\end{array}\right] \\
M^{3} & =\left[\begin{array}{cc}
w_{1} & 0 \\
0 & w_{2}
\end{array}\right] .
\end{aligned}
$$

First assume $c=2$. All three matrices exhibit generic behavior. The points in this orbit are folding generic. Since $2 c+3=7=2^{3}-1$, this 
is the generic orbit, and the points in this orbit are also rank generic. The generic rank is 2. Notice that some other orbits also consisted of elements of rank 2. Now assume $c>2$. $M^{1}$ and $M^{2}$ do not exhibit generic behavior because the dimension of the scheme defined by their $2 \times 2$ determinants is too large. It has dimension 0 whereas it should be empty. $M^{3}$ does not exhibit generic behavior because the scheme defined by its $2 \times 2$ minor is reducible. Also, the scheme defined by its $1 \times 1$ minors has too large a dimension. The points in this orbit are not folding generic.

Notice that orbits 7, 8 and 9 require $c>2$.

Orbit 7. This has dimension $3 c+1$ with typical element $u_{1} \otimes v_{1} \otimes$ $w_{1}+u_{1} \otimes v_{2} \otimes w_{3}+u_{2} \otimes v_{1} \otimes w_{2}$ of rank 3. $M^{1}$ is $2 \times c, M^{2}$ is $2 \times c$, and $M^{3}$ is $2 \times 2$.

$$
\begin{aligned}
M^{1} & =\left[\begin{array}{ccccc}
u_{1} & u_{2} & 0 & 0 & \ldots \\
0 & 0 & u_{1} & 0 & \ldots
\end{array}\right] \\
M^{2} & =\left[\begin{array}{ccccc}
v_{1} & 0 & v_{2} & 0 & \ldots \\
0 & v_{1} & 0 & 0 & \ldots
\end{array}\right] \\
M^{3} & =\left[\begin{array}{cc}
w_{1} & w_{3} \\
w_{2} & 0
\end{array}\right] .
\end{aligned}
$$

$M^{1}$ and $M^{2}$ do not exhibit generic behavior because the scheme defined by their $2 \times 2$ minors has dimension too large. $M^{3}$ does not exhibit generic behavior because the scheme defined by its $2 \times 2$ minor is reducible. The points in this orbit are not folding generic.

Orbit 8. This has dimension $3 c+2$ with typical element $u_{1} \otimes v_{1} \otimes$ $w_{1}+u_{1} \otimes v_{2} \otimes w_{2}+u_{2} \otimes v_{1} \otimes w_{2}+u_{2} \otimes v_{2} \otimes w_{3}$ of rank 3. $M^{1}$ is $2 \times c$, $M^{2}$ is $2 \times c$ and $M^{3}$ is $2 \times 2$.

$$
\begin{aligned}
M^{1} & =\left[\begin{array}{ccccc}
u_{1} & u_{2} & 0 & 0 & \ldots \\
0 & u_{1} & u_{2} & 0 & \ldots
\end{array}\right] \\
M^{2} & =\left[\begin{array}{lllll}
v_{1} & v_{2} & 0 & 0 & \ldots \\
0 & v_{1} & v_{2} & 0 & \ldots
\end{array}\right] \\
M^{3} & =\left[\begin{array}{ll}
w_{1} & w_{2} \\
w_{2} & w_{3}
\end{array}\right] .
\end{aligned}
$$

$M^{1}$ and $M^{2}$ exhibit generic behavior. The schemes defined by both their $2 \times 2$ and $1 \times 1$ determinants are empty as they should be. Now assume $c=3$. $M^{3}$ exhibits generic behavior. Points in this orbit are 
folding generic. Since $3 c+2=11=(2)(2)(3)-1$, this is the generic orbit, and points in this orbit are also rank generic. Finally, assume $c>3 . M^{3}$ does not exhibit generic behavior as the dimension of the scheme defined by its $1 \times 1$ determinants is too large. The points in this orbit are not folding generic.

Notice that Orbit 9 requires $c>3$.

Orbit 9. This has dimension $4 c-1$ with typical element $u_{1} \otimes v_{1} \otimes$ $w_{1}+u_{1} \otimes v_{2} \otimes w_{3}+u_{2} \otimes v_{1} \otimes w_{2}+u_{2} \otimes v_{2} \otimes w_{4}$ of rank $4 . M^{1}$ is $2 \times c$, $M^{2}$ is $2 \times c$ and $M^{3}$ is $2 \times 2$.

$$
\begin{aligned}
M^{1} & =\left[\begin{array}{cccccc}
u_{1} & u_{2} & 0 & 0 & 0 & \ldots \\
0 & 0 & u_{1} & u_{2} & 0 & \ldots
\end{array}\right] \\
M^{2} & =\left[\begin{array}{cccccc}
v_{1} & 0 & v_{2} & 0 & 0 & \ldots \\
0 & v_{1} & 0 & v_{2} & 0 & \ldots
\end{array}\right] \\
M^{3} & =\left[\begin{array}{ll}
w_{1} & w_{3} \\
w_{2} & w_{4}
\end{array}\right] .
\end{aligned}
$$

All three matrices exhibit generic behavior. See Orbit 26 for a more complete explanation of an analogous situation. Points in this orbit are folding generic. As $4 c-1=(2)(2) c-1$, this is the generic orbit. Points in this orbit are also rank generic.

Now we proceed to the case where $V$ has dimension 3. Table 10.3 .2 in [18] describes how the first nine orbits carry over to this case. We may assume that $c \geq 3$ as the case where $c=2$ is equivalent to the case where $V$ has dimension 2 and $c=3$. None of the first nine orbits will be folding generic. The variable $v_{3}$ will never appear in $M^{2}$. The scheme defined by the $1 \times 1$ minors of $M^{2}$ will always have a dimension that is too large. We work through Orbits 10-26 as we did for Orbits 1-9 in the previous case. These orbits are described in [18, Tables 10.3.3-10.3.5].

Orbit 10. This has dimension $3 c$ with typical element $u_{1} \otimes v_{1} \otimes w_{1}+$ $u_{1} \otimes v_{2} \otimes w_{2}+u_{1} \otimes v_{3} \otimes w_{3}$ of rank 3. $M^{1}$ is $3 \times c, M^{2}$ is $2 \times c$ and 
$M^{3}$ is $2 \times 3$.

$$
\begin{aligned}
M^{1} & =\left[\begin{array}{ccccc}
u_{1} & 0 & 0 & 0 & \ldots \\
0 & u_{1} & 0 & 0 & \ldots \\
0 & 0 & u_{1} & 0 & \ldots
\end{array}\right] \\
M^{2} & =\left[\begin{array}{ccccc}
v_{1} & v_{2} & v_{3} & 0 & \ldots \\
0 & 0 & 0 & 0 & \ldots
\end{array}\right] \\
M^{3} & =\left[\begin{array}{ccc}
w_{1} & w_{2} & w_{3} \\
0 & 0 & 0
\end{array}\right] .
\end{aligned}
$$

$M^{1}$ does not exhibit generic behavior because its $3 \times 3$ determinants define a non-reduced scheme. $M^{2}$ and $M^{3}$ do not exhibit generic behavior as their $2 \times 2$ determinants are all 0 . The points in this orbit are not folding generic.

Orbit 11 . This has dimension $2 c+6$ with typical element $u_{1} \otimes v_{1} \otimes$ $w_{1}+u_{1} \otimes v_{3} \otimes w_{2}+u_{2} \otimes v_{2} \otimes w_{1}$ of rank 3. $M^{1}$ is $3 \times c, M^{2}$ is $2 \times c$ and $M^{3}$ is $2 \times 3$.

$$
\begin{aligned}
M^{1} & =\left[\begin{array}{ccccc}
u_{1} & 0 & 0 & 0 & \ldots \\
u_{2} & 0 & 0 & 0 & \ldots \\
0 & u_{1} & 0 & 0 & \ldots
\end{array}\right] \\
M^{2} & =\left[\begin{array}{ccccc}
v_{1} & v_{3} & 0 & 0 & \ldots \\
v_{2} & 0 & 0 & 0 & \ldots
\end{array}\right] \\
M^{3} & =\left[\begin{array}{ccc}
w_{1} & 0 & w_{2} \\
0 & w_{1} & 0
\end{array}\right] .
\end{aligned}
$$

$M^{1}$ does not exhibit generic behavior because its $3 \times 3$ determinants are all $0 . \quad M^{2}$ does not exhibit generic behavior because its $2 \times 2$ determinants give a scheme of too large dimension. $M^{3}$ does not exhibit generic behavior because its $1 \times 1$ determinants give a scheme of too large dimension. The points in this orbit are not folding generic.

Orbit 12 . This has dimension $2 c+7$ with typical element $u_{1} \otimes v_{1} \otimes$ $w_{1}+u_{1} \otimes v_{2} \otimes w_{2}+u_{2} \otimes v_{2} \otimes w_{1}+u_{2} \otimes v_{3} \otimes w_{2}$ of rank 3. $M^{1}$ is $3 \times c$, 
$M^{2}$ is $2 \times c$ and $M^{3}$ is $2 \times 3$.

$$
\begin{aligned}
M^{1} & =\left[\begin{array}{ccccc}
u_{1} & 0 & 0 & 0 & \ldots \\
u_{2} & u_{1} & 0 & 0 & \ldots \\
0 & u_{2} & 0 & 0 & \ldots
\end{array}\right] \\
M^{2} & =\left[\begin{array}{lllll}
v_{1} & v_{2} & 0 & 0 & \ldots \\
v_{2} & v_{3} & 0 & 0 & \ldots
\end{array}\right] \\
M^{3} & =\left[\begin{array}{ccc}
w_{1} & w_{2} & 0 \\
0 & w_{1} & w_{2}
\end{array}\right] .
\end{aligned}
$$

$M^{1}$ does not exhibit generic behavior because all its $3 \times 3$ determinants are $0 . M^{2}$ does not exhibit generic behavior because the scheme defined by its $2 \times 2$ determinants has dimension too large. $M^{3}$ does not exhibit generic behavior because the scheme defined by its $1 \times 1$ determinants has dimension too large. The points in this orbit are not folding generic.

Orbit 13. A typical element is $u_{1} \otimes v_{1} \otimes w_{1}+u_{1} \otimes v_{2} \otimes w_{3}+u_{2} \otimes$ $v_{1} \otimes w_{2}+u_{2} \otimes v_{3} \otimes w_{3}$ of rank 4. $M^{1}$ is $3 \times c, M^{2}$ is $2 \times c$ and $M^{3}$ is $2 \times 3$.

$$
\begin{aligned}
M^{1} & =\left[\begin{array}{ccccc}
u_{1} & u_{2} & 0 & 0 & \ldots \\
0 & 0 & u_{1} & 0 & \ldots \\
0 & 0 & u_{2} & 0 & \ldots
\end{array}\right] \\
M^{2} & =\left[\begin{array}{ccccc}
v_{1} & 0 & v_{2} & 0 & \ldots \\
0 & v_{1} & v_{3} & 0 & \ldots
\end{array}\right] \\
M^{3} & =\left[\begin{array}{ccc}
w_{1} & w_{3} & 0 \\
w_{2} & 0 & w_{3}
\end{array}\right] .
\end{aligned}
$$

$M^{1}$ does not exhibit generic behavior because all its $3 \times 3$ determinants are $0 . M^{2}$ and $M^{3}$ do not exhibit generic behavior because the scheme determined by their $2 \times 2$ determinants has dimension too large. The points in this orbit are not folding generic.

Orbit 14. A typical element is $u_{1} \otimes v_{1} \otimes w_{1}+u_{1} \otimes v_{2} \otimes w_{2}+u_{2} \otimes v_{3} \otimes w_{3}$ 
of rank 3. $M^{1}$ is $3 \times c, M^{2}$ is $2 \times c$ and $M^{3}$ is $2 \times 3$.

$$
\begin{aligned}
M^{1} & =\left[\begin{array}{ccccc}
u_{1} & 0 & 0 & 0 & \ldots \\
0 & u_{1} & 0 & 0 & \ldots \\
0 & 0 & u_{2} & 0 & \ldots
\end{array}\right] \\
M^{2} & =\left[\begin{array}{ccccc}
v_{1} & v_{2} & 0 & 0 & \ldots \\
0 & 0 & v_{3} & 0 & \ldots
\end{array}\right] \\
M^{3} & =\left[\begin{array}{ccc}
w_{1} & w_{2} & 0 \\
0 & 0 & w_{3}
\end{array}\right] .
\end{aligned}
$$

$M^{1}$ does not exhibit generic behavior because the scheme defined by its $3 \times 3$ determinants is not reduced. $M^{2}$ and $M^{3}$ do not exhibit generic behavior because the scheme defined by their $2 \times 2$ determinants has dimension too large. The points in this orbit are not folding generic.

Orbit 15. A typical element is $u_{1} \otimes v_{1} \otimes w_{1}+u_{1} \otimes v_{2} \otimes w_{2}+u_{1} \otimes$ $v_{3} \otimes w_{3}+u_{2} \otimes v_{1} \otimes w_{2}$ of rank $4 . M^{1}$ is $3 \times c, M^{2}$ is $2 \times c$ and $M^{3}$ is $2 \times 3$.

$$
\begin{aligned}
M^{1} & =\left[\begin{array}{ccccc}
u_{1} & u_{2} & 0 & 0 & \ldots \\
0 & u_{1} & 0 & 0 & \ldots \\
0 & 0 & u_{1} & 0 & \ldots
\end{array}\right] \\
M^{2} & =\left[\begin{array}{ccccc}
v_{1} & v_{2} & v_{3} & 0 & \ldots \\
0 & v_{1} & 0 & 0 & \ldots
\end{array}\right] \\
M^{3} & =\left[\begin{array}{ccc}
w_{1} & w_{2} & w_{3} \\
w_{2} & 0 & 0
\end{array}\right] .
\end{aligned}
$$

$M^{1}$ does not exhibit generic behavior because the scheme defined by its $3 \times 3$ determinants is not reduced. $M^{2}$ and $M^{3}$ do not exhibit generic behavior because the scheme defined by their $2 \times 2$ determinants has dimension too large. The points in this orbit are not folding generic.

Orbit 16. A typical element is $u_{1} \otimes v_{1} \otimes w_{1}+u_{1} \otimes v_{2} \otimes w_{2}+u_{1} \otimes$ $v_{3} \otimes w_{3}+u_{2} \otimes v_{1} \otimes w_{2}+u_{2} \otimes v_{2} \otimes w_{3}$ of rank 4. $M^{1}$ is $3 \times c, M^{2}$ is 
$2 \times c$ and $M^{3}$ is $2 \times 3$.

$$
\begin{aligned}
M^{1} & =\left[\begin{array}{ccccc}
u_{1} & u_{2} & 0 & 0 & \ldots \\
0 & u_{1} & u_{2} & 0 & \ldots \\
0 & 0 & u_{1} & 0 & \ldots
\end{array}\right] \\
M^{2} & =\left[\begin{array}{lllll}
v_{1} & v_{2} & v_{3} & 0 & \ldots \\
0 & v_{1} & v_{2} & 0 & \ldots
\end{array}\right] \\
M^{3} & =\left[\begin{array}{lll}
w_{1} & w_{2} & w_{3} \\
w_{2} & w_{3} & 0
\end{array}\right] .
\end{aligned}
$$

$M^{1}$ does not exhibit generic behavior because the scheme defined by its $3 \times 3$ determinants is not reduced. $M^{2}$ and $M^{3}$ do not exhibit generic behavior because the scheme defined by their $2 \times 2$ determinants is not reduced. The points in this orbit are not folding generic.

Orbit 17. This has dimension $3 c+7$ with typical element $u_{1} \otimes v_{1} \otimes$ $w_{1}+u_{1} \otimes v_{2} \otimes w_{2}+u_{2} \otimes v_{1} \otimes w_{2}+u_{2} \otimes v_{3} \otimes w_{3}$ of rank 4. $M^{1}$ is $3 \times c$, $M^{2}$ is $2 \times c$ and $M^{3}$ is $2 \times 3$.

$$
\begin{aligned}
M^{1} & =\left[\begin{array}{ccccc}
u_{1} & u_{2} & 0 & 0 & \ldots \\
0 & u_{1} & 0 & 0 & \ldots \\
0 & 0 & u_{2} & 0 & \ldots
\end{array}\right] \\
M^{2} & =\left[\begin{array}{ccccc}
v_{1} & v_{2} & 0 & 0 & \ldots \\
0 & v_{1} & v_{3} & 0 & \ldots
\end{array}\right] \\
M^{3} & =\left[\begin{array}{ccc}
w_{1} & w_{2} & 0 \\
w_{2} & 0 & w_{3}
\end{array}\right] .
\end{aligned}
$$

$M^{1}$ does not exhibit generic behavior because the scheme defined by its $3 \times 3$ determinants is not reduced. $M^{2}$ and $M^{3}$ do not exhibit generic behavior because the scheme defined by their $2 \times 2$ determinants is not reduced. The points in this orbit are not folding generic.

Orbit 18. This has dimension $3 c+8$ with typical element $u_{1} \otimes v_{1} \otimes$ $w_{1}+u_{1} \otimes v_{2} \otimes w_{2}+u_{2} \otimes v_{2} \otimes w_{2}+u_{2} \otimes v_{3} \otimes w_{3}$ of rank 3. $M^{1}$ is $3 \times c$, 
$M^{2}$ is $2 \times c$ and $M^{3}$ is $2 \times 3$.

$$
\begin{aligned}
M^{1} & =\left[\begin{array}{ccccc}
u_{1} & 0 & 0 & 0 & \ldots \\
0 & u_{1}+u_{2} & 0 & 0 & \ldots \\
0 & 0 & u_{2} & 0 & \ldots
\end{array}\right] \\
M^{2} & =\left[\begin{array}{ccccc}
v_{1} & v_{2} & 0 & 0 & \ldots \\
0 & v_{2} & v_{3} & 0 & \ldots
\end{array}\right] \\
M^{3} & =\left[\begin{array}{ccc}
w_{1} & w_{2} & 0 \\
0 & w_{2} & w_{3}
\end{array}\right] .
\end{aligned}
$$

First assume that $c=3$. All three matrices exhibit generic behavior. For $M^{1}$, the scheme defined by the $3 \times 3$ determinant consists of the 3 reduced points $[1,0],[0,1]$ and $[1,-1]$. The schemes defined by the $2 \times 2$ and $1 \times 1$ determinants are empty. For $M^{2}$ and $M^{3}$, the scheme defined by the $2 \times 2$ determinants consists of the three reduced points $[1,0,0],[0,1,0]$ and $[0,0,1]$. The scheme defined by the $1 \times 1$ determinants is empty. The points in this orbit are folding generic. As $(3)(3)+8=17=(2)(3)(3)-1$, this is the generic orbit, and points in this orbit are also rank generic. Now assume $c>3 . M^{1}$ does not exhibit generic behavior because the scheme defined by its $3 \times 3$ determinants has dimension too large. $M^{2}$ does not exhibit generic behavior because the scheme defined by its $2 \times 2$ determinants has dimension too large. $M^{3}$ does not exhibit generic behavior because the scheme defined by its $1 \times 1$ determinants has dimension too large. The points in this orbit are not folding generic.

Notice that orbits 19-23 require $c>3$.

Orbit 19. A typical element is $u_{1} \otimes v_{1} \otimes w_{1}+u_{1} \otimes v_{2} \otimes w_{2}+u_{1} \otimes$ $v_{3} \otimes w_{4}+u_{2} \otimes v_{1} \otimes w_{2}+u_{2} \otimes v_{2} \otimes w_{3}$ of rank 4 . $M^{1}$ is $3 \times c, M^{2}$ is $2 \times c$ and $M^{3}$ is $2 \times 3$.

$$
\begin{aligned}
M^{1} & =\left[\begin{array}{cccccc}
u_{1} & u_{2} & 0 & 0 & 0 & \ldots \\
0 & u_{1} & u_{2} & 0 & 0 & \ldots \\
0 & 0 & 0 & u_{1} & 0 & \ldots
\end{array}\right] \\
M^{2} & =\left[\begin{array}{cccccc}
v_{1} & v_{2} & 0 & v_{3} & 0 & \ldots \\
0 & v_{1} & v_{2} & 0 & 0 & \ldots
\end{array}\right] \\
M^{3} & =\left[\begin{array}{lll}
w_{1} & w_{2} & w_{4} \\
w_{2} & w_{3} & 0
\end{array}\right] .
\end{aligned}
$$

$M^{1}$ does not exhibit generic behavior because the scheme defined by 
its $3 \times 3$ determinants has dimension too large. $M^{2}$ does not exhibit generic behavior because the scheme defined by its $2 \times 2$ determinants has dimension too large. $M^{3}$ does not exhibit generic behavior because the scheme defined by its $2 \times 2$ determinants is reducible. The points in this orbit are not folding generic.

Orbit 20. A typical element is $u_{1} \otimes v_{1} \otimes w_{1}+u_{1} \otimes v_{2} \otimes w_{3}+u_{1} \otimes$ $v_{3} \otimes w_{4}+u_{2} \otimes v_{1} \otimes w_{2}$ of rank $4 . M^{1}$ is $3 \times c, M^{2}$ is $2 \times c$ and $M^{3}$ is $2 \times 3$.

$$
\begin{aligned}
M^{1} & =\left[\begin{array}{cccccc}
u_{1} & u_{2} & 0 & 0 & 0 & \ldots \\
0 & 0 & u_{1} & 0 & 0 & \ldots \\
0 & 0 & 0 & u_{1} & 0 & \ldots
\end{array}\right] \\
M^{2} & =\left[\begin{array}{cccccc}
v_{1} & 0 & v_{2} & v_{3} & 0 & \ldots \\
0 & v_{1} & 0 & 0 & 0 & \ldots
\end{array}\right] \\
M^{3} & =\left[\begin{array}{ccc}
w_{1} & w_{3} & w_{4} \\
w_{2} & 0 & 0
\end{array}\right] .
\end{aligned}
$$

$M^{1}$ does not exhibit generic behavior because the scheme defined by its $3 \times 3$ determinants has dimension too large. $M^{2}$ and $M^{3}$ do not exhibit generic behavior because the scheme defined by their $2 \times 2$ determinants has dimension too large. The points in this orbit are not folding generic.

Orbit 21. A typical element is $u_{1} \otimes v_{1} \otimes w_{1}+u_{1} \otimes v_{2} \otimes w_{3}+u_{1} \otimes$ $v_{3} \otimes w_{4}+u_{2} \otimes v_{1} \otimes w_{2}+u_{2} \otimes v_{2} \otimes w_{4}$ of rank 5. $M^{1}$ is $3 \times c, M^{2}$ is $2 \times c$ and $M^{3}$ is $2 \times 3$.

$$
\begin{aligned}
M^{1} & =\left[\begin{array}{cccccc}
u_{1} & u_{2} & 0 & 0 & 0 & \ldots \\
0 & 0 & u_{1} & u_{2} & 0 & \ldots \\
0 & 0 & 0 & u_{1} & 0 & \ldots
\end{array}\right] \\
M^{2} & =\left[\begin{array}{cccccc}
v_{1} & 0 & v_{2} & v_{3} & 0 & \ldots \\
0 & v_{1} & 0 & v_{2} & 0 & \ldots
\end{array}\right] \\
M^{3} & =\left[\begin{array}{lll}
w_{1} & w_{3} & w_{4} \\
w_{2} & w_{4} & 0
\end{array}\right] .
\end{aligned}
$$

$M^{1}$ does not exhibit generic behavior because the scheme defined by its $3 \times 3$ determinants has dimension too large. $M^{2}$ does not exhibit generic behavior because the scheme defined by its $2 \times 2$ determinants has dimension too large. $M^{3}$ does not exhibit generic behavior because 
the scheme defined by its $2 \times 2$ determinants is reducible. The points in this orbit are not folding generic.

Orbit 22. This has dimension $4 c+6$ with typical element $u_{1} \otimes v_{1} \otimes$ $w_{1}+u_{1} \otimes v_{2} \otimes w_{3}+u_{2} \otimes v_{1} \otimes w_{2}+u_{2} \otimes v_{3} \otimes w_{4}$ of rank $4 . M^{1}$ is $3 \times c$, $M^{2}$ is $2 \times c$ and $M^{3}$ is $2 \times 3$.

$$
\begin{aligned}
M^{1} & =\left[\begin{array}{cccccc}
u_{1} & u_{2} & 0 & 0 & 0 & \ldots \\
0 & 0 & u_{1} & 0 & 0 & \ldots \\
0 & 0 & 0 & u_{2} & 0 & \ldots
\end{array}\right] \\
M^{2} & =\left[\begin{array}{cccccc}
v_{1} & 0 & v_{2} & 0 & 0 & \ldots \\
0 & v_{1} & 0 & v_{3} & 0 & \ldots
\end{array}\right] \\
M^{3} & =\left[\begin{array}{ccc}
w_{1} & w_{3} & 0 \\
w_{2} & 0 & w_{4}
\end{array}\right] .
\end{aligned}
$$

$M^{1}$ does not exhibit generic behavior because the scheme defined by its $3 \times 3$ determinants has dimension too large. $M^{2}$ does not exhibit generic behavior because the scheme defined by its $2 \times 2$ determinants has dimension too large. $M^{3}$ does not exhibit generic behavior because the scheme defined by its $2 \times 2$ determinants is reducible. The points in this orbit are not folding generic.

Orbit 23. This has dimension $4 c+7$ with typical element $u_{1} \otimes v_{1} \otimes$ $w_{1}+u_{1} \otimes v_{2} \otimes w_{2}+u_{1} \otimes v_{3} \otimes w_{3}+u_{2} \otimes v_{1} \otimes w_{2}+u_{2} \otimes v_{2} \otimes w_{3}+u_{2} \otimes v_{3} \otimes w_{4}$ of rank 4. $M^{1}$ is $3 \times c, M^{2}$ is $2 \times c$ and $M^{3}$ is $2 \times 3$.

$$
\begin{aligned}
M^{1} & =\left[\begin{array}{cccccc}
u_{1} & u_{2} & 0 & 0 & 0 & \ldots \\
0 & u_{1} & u_{2} & 0 & 0 & \ldots \\
0 & 0 & u_{1} & u_{2} & 0 & \ldots
\end{array}\right] \\
M^{2} & =\left[\begin{array}{llllll}
v_{1} & v_{2} & v_{3} & 0 & 0 & \ldots \\
0 & v_{1} & v_{2} & v_{3} & 0 & \ldots
\end{array}\right] \\
M^{3} & =\left[\begin{array}{lll}
w_{1} & w_{2} & w_{3} \\
w_{2} & w_{3} & w_{4}
\end{array}\right] .
\end{aligned}
$$

$M^{1}$ and $M^{2}$ exhibit generic behavior because all the determinantal schemes associated to them are empty.

Now assume $c=4 . \quad M^{3}$ exhibits generic behavior. The scheme defined by its $2 \times 2$ determinants is the twisted cubic curve in $\mathbb{P}^{3},[\mathbf{1 2}$, Example 1.10], and the scheme defined by its $1 \times 1$ determinants is empty. The points in this orbit are folding generic. As $(4)(4)+7=$ 
$23=(2)(3)(4)-1$, this is the generic orbit. Points in this orbit are rank generic. Finally, assume $c>4 . M^{3}$ does not exhibit generic behavior because the scheme defined by its $1 \times 1$ minors has dimension too large. Points in this orbit are not folding generic.

Notice that orbits 24 and 25 require $c>4$.

Orbit 24. This has dimension $5 c$ with typical element $u_{1} \otimes v_{1} \otimes w_{1}+$ $u_{1} \otimes v_{2} \otimes w_{3}+u_{1} \otimes v_{3} \otimes w_{5}+u_{2} \otimes v_{1} \otimes w_{2}+u_{2} \otimes v_{2} \otimes w_{4}$ of rank 5 . $M^{1}$ is $3 \times c, M^{2}$ is $2 \times c$ and $M^{3}$ is $2 \times 3$.

$$
\begin{aligned}
M^{1} & =\left[\begin{array}{ccccccc}
u_{1} & u_{2} & 0 & 0 & 0 & 0 & \ldots \\
0 & 0 & u_{1} & u_{2} & 0 & 0 & \ldots \\
0 & 0 & 0 & 0 & u_{1} & 0 & \ldots
\end{array}\right] \\
M^{2} & =\left[\begin{array}{ccccccc}
v_{1} & 0 & v_{2} & 0 & v_{3} & 0 & \ldots \\
0 & v_{1} & 0 & v_{2} & 0 & 0 & \ldots
\end{array}\right] \\
M^{3} & =\left[\begin{array}{lll}
w_{1} & w_{3} & w_{5} \\
w_{2} & w_{4} & 0
\end{array}\right] .
\end{aligned}
$$

$M^{1}$ does not exhibit generic behavior because the scheme defined by its $3 \times 3$ determinants has dimension too large. $M^{2}$ does not exhibit generic behavior because the scheme defined by its $2 \times 2$ determinants has dimension too large. $M^{3}$ does not exhibit generic behavior because the scheme defined by its $2 \times 2$ determinants is reducible. Points in this orbit are not folding generic.

Orbit 25. This has dimension $5 c+4$ with typical element $u_{1} \otimes v_{1} \otimes$ $w_{1}+u_{1} \otimes v_{2} \otimes w_{2}+u_{1} \otimes v_{3} \otimes w_{4}+u_{2} \otimes v_{1} \otimes w_{2}+u_{2} \otimes v_{2} \otimes w_{3}+u_{2} \otimes v_{3} \otimes w_{5}$ of rank $5 . M^{1}$ is $3 \times c, M^{2}$ is $2 \times c$ and $M^{3}$ is $2 \times 3$.

$$
\begin{aligned}
M^{1} & =\left[\begin{array}{ccccccc}
u_{1} & u_{2} & 0 & 0 & 0 & 0 & \ldots \\
0 & u_{1} & u_{2} & 0 & 0 & 0 & \ldots \\
0 & 0 & 0 & u_{1} & u_{2} & 0 & \ldots
\end{array}\right] \\
M^{2} & =\left[\begin{array}{lllllll}
v_{1} & v_{2} & 0 & v_{3} & 0 & 0 & \ldots \\
0 & v_{1} & v_{2} & 0 & v_{3} & 0 & \ldots
\end{array}\right] \\
M^{3} & =\left[\begin{array}{lll}
w_{1} & w_{2} & w_{4} \\
w_{2} & w_{3} & w_{5}
\end{array}\right] .
\end{aligned}
$$

$M^{1}$ and $M^{2}$ exhibit generic behavior as all their determinantal schemes are empty.

Now assume $c=5$. The three $2 \times 2$ determinants of $M^{3}$ are: 
$w_{1} w_{3}-w_{2}^{2}, w_{2} w_{5}-w_{3} w_{4}, w_{1} w_{5}-w_{2} w_{4}$. Their common zeros are the closure of the image of the rational map $\mathbb{P}^{2} \rightarrow \mathbb{P}^{4}$ given by $[x, y, z] \rightarrow\left[z^{2}, x z, x^{2}, y z, x y\right]$. This is a nonsingular surface, see [3, Chapter IV]. One can also check nonsingularity, hence reducedness, by looking at the rank of the matrix of partial derivatives of the three equations.

$$
N=\left[\begin{array}{ccccc}
w_{3} & -2 w_{2} & w_{1} & 0 & 0 \\
0 & w_{5} & -w_{4} & -w_{3} & w_{2} \\
w_{5} & -w_{4} & 0 & -w_{2} & w_{1}
\end{array}\right]
$$

A singular point would be a point on the surface where $N$ has rank less than 2. That is where all the $2 \times 2$ determinants of $N$ vanish. Looking at rows 2 and 3 and columns 1 and 2 we get $w_{5}=0$. Looking at rows 1 and 2 and columns 1 and 4 we get $w_{3}=0$. Putting this into the equation $w_{1} w_{3}-w_{2}^{2}=0$, we get $w_{2}=0$. Putting this into $N$, it becomes

$$
N=\left[\begin{array}{ccccc}
0 & 0 & w_{1} & 0 & 0 \\
0 & 0 & -w_{4} & 0 & 0 \\
0 & -w_{4} & 0 & 0 & w_{1}
\end{array}\right] .
$$

The only way for this to have rank less than 2 is for $w_{1}=w_{4}=0$. Now all five coordinates are 0 , so there are no such points. The scheme defined by the $1 \times 1$ determinants of $M^{3}$ is empty. $M^{3}$ exhibits generic behavior. The points in this orbit are folding generic. As $(5)(5)+4=29=(2)(3)(5)-1$, this is the generic orbit, and points in this orbit are rank generic.

Next, assume $c>5 . \quad M^{3}$ does not exhibit generic behavior. The scheme defined by its $1 \times 1$ determinants has dimension too large. The points in this orbit are not folding generic.

Notice that orbit 26 requires $c>5$.

Orbit 26. This has dimension $6 c-1$ with typical element $u_{1} \otimes v_{1} \otimes$ $w_{1}+u_{1} \otimes v_{2} \otimes w_{3}+u_{1} \otimes v_{3} \otimes w_{5}+u_{2} \otimes v_{1} \otimes w_{2}+u_{2} \otimes v_{2} \otimes w_{4}+u_{2} \otimes v_{3} \otimes w_{6}$ 
of rank $6 . M^{1}$ is $3 \times c, M^{2}$ is $2 \times c$ and $M^{3}$ is $2 \times 3$.

$$
\begin{aligned}
M^{1} & =\left[\begin{array}{cccccccc}
u_{1} & u_{2} & 0 & 0 & 0 & 0 & 0 & \ldots \\
0 & 0 & u_{1} & u_{2} & 0 & 0 & 0 & \ldots \\
0 & 0 & 0 & 0 & u_{1} & u_{2} & 0 & \ldots
\end{array}\right] \\
M^{2} & =\left[\begin{array}{cccccccc}
v_{1} & 0 & v_{2} & 0 & v_{3} & 0 & 0 & \ldots \\
0 & v_{1} & 0 & v_{2} & 0 & v_{3} & 0 & \ldots
\end{array}\right] \\
M^{3} & =\left[\begin{array}{llll}
w_{1} & w_{3} & w_{5} \\
w_{2} & w_{4} & w_{6}
\end{array}\right] .
\end{aligned}
$$

$M^{1}$ and $M^{2}$ exhibit generic behavior as all their determinantal schemes are empty. As for $M^{3}$, when $c=6$, this is a generic matrix (all six entries are different variables), so it certainly exhibits generic behavior. When $c>6$ for $2 \times 2$ determinants, the schemes are cones over the $2 \times 2$ locus when $c=6$, which gives them the correct dimension. Their singular locus is the vertex of the cone, which is when $w_{1}=w_{2}=w_{3}=w_{4}=w_{5}=w_{6}=0$, that is, the scheme defined by the $1 \times 1$ determinants as required. The scheme defined by the $1 \times 1$ determinants is a linear variety of dimension $c-7$, which meets all generic requirements. Points in this orbit are folding generic. As $6 c-1=(2)(3) c-1$, this is the generic orbit, and points in this orbit are rank generic.

Example 4.8. In this example, we show that there do exist folding generic tensors that are not rank generic. It is based on ideas suggested by the referee. Consider $V \otimes \cdots \otimes V=V^{\otimes n}$. There is a natural action of the symmetric group $S_{n}$ on $V^{\otimes n}$ which simply permutes the factors. A tensor $T \in V^{\otimes n}$ is called symmetric if and only if $\sigma T=T$ for all $\sigma \in S_{n}$. If $T$ is symmetric, we can define the symmetric rank of $T$ to be the smallest number of simple tensors in an expression of $T$ as a sum of symmetric simple tensors. It is obvious that the rank of a symmetric tensor is less than or equal to its symmetric rank. See [18] for more information on symmetric tensors.

Now we restrict to the case of $\mathbb{C}^{3} \otimes \mathbb{C}^{3} \otimes \mathbb{C}^{3}$. Let $\left\{x_{1}, x_{2}, x_{3}\right\}$ be a basis for $\mathbb{C}^{3}$. Let

$$
\begin{aligned}
T= & x_{1} \otimes x_{1} \otimes x_{1}-x_{1} \otimes x_{3} \otimes x_{3}-x_{3} \otimes x_{1} \otimes x_{3}-x_{3} \otimes x_{3} \otimes x_{1} \\
& +x_{2} \otimes x_{2} \otimes x_{3}+x_{2} \otimes x_{3} \otimes x_{2}+x_{3} \otimes x_{2} \otimes x_{2} .
\end{aligned}
$$


Notice that $T$ is symmetric. We now check that $T$ is folding generic. We will abuse our notation using the $x_{i}$ 's to also denote variables. All three foldings of $T$ equal:

$$
\left[\begin{array}{ccc}
x_{1} & 0 & -x_{3} \\
0 & x_{3} & x_{2} \\
-x_{3} & x_{2} & -x_{1}
\end{array}\right] .
$$

Clearly, the set of common zeroes of the $1 \times 1$ subdeterminants is empty. The nine $2 \times 2$ subdeterminants are $x_{1} x_{3}, x_{3}^{2}, x_{1} x_{2}, x_{3}^{2}$, $-x_{1} x_{3}-x_{2}^{2}, x_{2} x_{3}, x_{1} x_{2}, x_{2} x_{3},-x_{1}^{2}-x_{3}^{2}$. Polynomials $x_{3}^{2},-x_{1} x_{3}-x_{2}^{2}$ and $-x_{1}^{2}-x_{3}^{2}$ already have empty common zeroes. The $3 \times 3$ determinant is $-x_{1}^{2} x_{3}-x_{1} x_{2}^{2}-x_{3}^{3}$. Taking all three partial derivatives one infers that this is a non-singular plane cubic. It follows that a generic symmetric tensor in $\mathbb{C}^{3} \otimes \mathbb{C}^{3} \otimes \mathbb{C}^{3}$ is folding generic. From a result of Strassen (see [18, Theorem 3.1.4.3 (1)], a generic element of $\mathbb{C}^{3} \otimes \mathbb{C}^{3} \otimes \mathbb{C}^{3}$ has rank 5. From a result of Alexander-Hirschowitz (see [1] or [18, Theorem 3.2.2.4]) a generic symmetric element of $\mathbb{C}^{3} \otimes \mathbb{C}^{3} \otimes \mathbb{C}^{3}$ has symmetric rank 4 and thus rank at most 4 . Thus, a generic symmetric element of $\mathbb{C}^{3} \otimes \mathbb{C}^{3} \otimes \mathbb{C}^{3}$ is folding generic but not rank generic.

Remark 4.9. We think it would be interesting to explore further the question of in what generality the statement that folding generic implies rank generic remains true. As the preceding example shows this is not always the case. Notice the statement makes sense not just for three tensors, but tensors of higher order also.

5. The elasticity tensor. In previous sections, our examples of tensors were artificially created to have specific rank properties. Below we analyze the fourth order elasticity tensor relevant in mechanics. We compute the multilinear rank, size 2 multiplex rank and the folding rank of the elasticity tensor concluding that its rank is at least 9 and then further showing that it is not folding generic. Partial differential equations of linear elasticity are satisfied by a vector field of infinitesimal displacements of the points in a body. If a body is made of homogeneous, isotropic material those equations involve a fourth-order tensor $T$, see $[\mathbf{6}, \mathbf{1 9}]$, which we specify below.

Consider four vector spaces $U, V, W$ and $X$ with bases $\left\{u_{1}, u_{2}, u_{3}\right\}$, 
$\left\{v_{1}, v_{2}, v_{3}\right\},\left\{w_{1}, w_{2}, w_{3}\right\}$ and $\left\{x_{1}, x_{2}, x_{3}\right\}$. Consider $T \in U \otimes V \otimes W \otimes X$, $T=\sum_{i=1}^{3} \sum_{j=1}^{3}\left(\lambda u_{i} \otimes v_{i} \otimes w_{j} \otimes x_{j}+\mu\left(u_{i} \otimes v_{j} \otimes w_{i} \otimes x_{j}+u_{i} \otimes v_{j} \otimes w_{j} \otimes x_{i}\right)\right)$,

for some fixed positive constants $\lambda$ and $\mu$, called Lamé moduli. Typically, the elasticity tensor $T$ is treated as a tensor over the real numbers; however, our analysis requires that we view it as a complex tensor.

First, we compute the multilinear rank of $T$. The image of $T_{1}\left(U^{*}\right)$ is spanned by $\left\{\sum_{j=1}^{3}\left(\lambda v_{i} \otimes w_{j} \otimes x_{j}+\mu\left(v_{j} \otimes w_{i} \otimes x_{j}+v_{j} \otimes w_{j} \otimes x_{i}\right)\right)\right\}_{i=1}^{3}$. In coordinate form, each of these is a $3 \times 3 \times 3$ array of numbers which, for compactness, we list as a 27-tuple in lexicographic order

$$
\begin{gathered}
(\lambda+2 \mu, 0,0,0, \lambda, 0,0,0, \lambda, 0, \mu, 0, \mu, 0,0,0,0,0,0,0, \mu, 0,0,0, \mu, 0,0) \\
(0, \mu, 0, \mu, 0,0,0,0,0, \lambda, 0,0,0, \lambda+2 \mu, 0,0,0, \lambda, 0,0,0,0,0, \mu, 0, \mu, 0) \\
(0,0, \mu, 0,0,0, \mu, 0,0,0,0,0,0,0, \mu, 0, \mu, 0, \lambda, 0,0,0, \lambda, 0,0,0, \lambda+2 \mu) .
\end{gathered}
$$

These are obviously linearly independent. The other components of multilinear rank proceed similarly. Thus,

$$
\operatorname{rank}_{1}(T)=(3,3,3,3) .
$$

We conclude that $\operatorname{rank}(T) \geq 3$.

Next, we compute the size 2 multiplex rank and the folding rank of $T$. As we have seen, they are related so we compute them together. To compute components of either, one partitions $\{1,2,3,4\}=A \cup A^{\prime}$ into two sets of size 2 . There are six ways to do this. Looking at symmetries in $T$ one sees that there are only two essentially different ways of doing it, $A=\{1,2\}$ and $\{3,4\}$ behave similarly to each other and the other four choices for $A$ behave similarly to each other. For $A=\{1,2\}$, the folded matrix is $M=\left[m_{i, j}\right]$, where for $i \neq j, m_{i, j}=\mu\left(w_{i} x_{j}+w_{j} x_{i}\right)$ and $m_{i, i}=\lambda\left(w_{1} x_{1}+w_{2} x_{2}+w_{3} x_{3}\right)+2 \mu w_{i} x_{i}$. For $A=\{1,3\}$, the folded matrix is $N=\left[n_{i, j}\right]$, where for $i \neq j, n_{i, j}=\lambda v_{i} x_{j}+\mu v_{j} x_{i}$ and $n_{i, i}=\mu\left(v_{1} x_{1}+v_{2} x_{2}+v_{3} x_{3}\right)+(\lambda+\mu) v_{i} x_{i}$. Both $M$ and $N$ have rank 3 , so the

$$
\operatorname{rankf}(T)=(3,3,3,3,3,3) .
$$

Again, we conclude that $\operatorname{rank},(T) \geq 3$. To compute the size 2 multiplex rank of $T$ we need to compute the dimension of the linear span of all 
matrices $M$ for all specific values of the variables $w_{i}$ and $x_{j}$ and then do the same for $N$.

$M$ is symmetric, so its space will have dimension at most 6 . In fact, it is 6 as can be seen by the following six choices of specific values for the variables and the resulting matrices. In each, we set two of the variables equal to 1 . The others are set to $0 . w_{1}=x_{1}=1, w_{2}=x_{2}=1$, $w_{3}=x_{3}=1, w_{1}=x_{2}=1, w_{1}=x_{3}=1, w_{2}=x_{3}=1$. The resulting matrices are:

$$
\begin{gathered}
{\left[\begin{array}{ccc}
\lambda+2 \mu & 0 & 0 \\
0 & \lambda & 0 \\
0 & 0 & \lambda
\end{array}\right], \quad\left[\begin{array}{ccc}
\lambda & 0 \\
0 & \lambda+2 \mu & 0 \\
0 & 0 & \lambda
\end{array}\right], \quad\left[\begin{array}{ccc}
\lambda & 0 & 0 \\
0 & \lambda & 0 \\
0 & 0 & \lambda+2 \mu
\end{array}\right],} \\
{\left[\begin{array}{ccc}
0 & \mu & 0 \\
\mu & 0 & 0 \\
0 & 0 & 0
\end{array}\right], \quad\left[\begin{array}{lll}
0 & 0 & \mu \\
0 & 0 & 0 \\
\mu & 0 & 0
\end{array}\right], \quad\left[\begin{array}{lll}
0 & 0 & 0 \\
0 & 0 & \mu \\
0 & \mu & 0
\end{array}\right] .}
\end{gathered}
$$

These are easily seen to be linearly independent.

When $\lambda=\mu, N$ is symmetric so its space has dimension at most 6. When $\lambda \neq \mu, N$ is not symmetric so its space could perhaps have dimension as large as 9 . Both these upper bounds are achieved as can be seen by the following nine choices of specific values for the variables and the resulting matrices. In each, we set two of the variables equal to 1 . The others are set to $0 . v_{1}=x_{1}=1, v_{2}=x_{2}=1, v_{3}=x_{3}=1$, $v_{1}=x_{2}=1, v_{2}=x_{1}=1, v_{1}=x_{3}=1, v_{3}=x_{1}=1, v_{2}=x_{3}=1$ and $v_{3}=x_{2}=1$. The resulting matrices are:

$$
\begin{gathered}
{\left[\begin{array}{ccc}
\lambda+2 \mu & 0 & 0 \\
0 & \mu & 0 \\
0 & 0 & \mu
\end{array}\right],\left[\begin{array}{ccc}
\mu & 0 & 0 \\
0 & \lambda+2 \mu & 0 \\
0 & 0 & \mu
\end{array}\right],\left[\begin{array}{ccc}
\mu & 0 & 0 \\
0 & \mu & 0 \\
0 & 0 & \lambda+2 \mu
\end{array}\right],\left[\begin{array}{ccc}
0 & \lambda & 0 \\
\mu & 0 & 0 \\
0 & 0 & 0
\end{array}\right],} \\
{\left[\begin{array}{lll}
0 & \mu & 0 \\
\lambda & 0 & 0 \\
0 & 0 & 0
\end{array}\right],\left[\begin{array}{lll}
0 & 0 & \lambda \\
0 & 0 & 0 \\
\mu & 0 & 0
\end{array}\right],\left[\begin{array}{lll}
0 & 0 & \mu \\
0 & 0 & 0 \\
\lambda & 0 & 0
\end{array}\right],\left[\begin{array}{lll}
0 & 0 & 0 \\
0 & 0 & \lambda \\
0 & \mu & 0
\end{array}\right],\left[\begin{array}{lll}
0 & 0 & 0 \\
0 & 0 & \mu \\
0 & \lambda & 0
\end{array}\right]}
\end{gathered}
$$

When $\lambda=\mu$, these span a six-dimensional space, and thus $\operatorname{rank}_{2}(T)=$ $(6,6,6,6,6,6)$. We conclude that the rank of $T$ is at least 6 . When $\lambda \neq \mu$, these span a nine-dimensional space. This shows that

$$
\operatorname{rank}_{2}(T)=(6,9,9,9,9,6) .
$$


We conclude that the rank of $T$ is at least 9 . Notice that size 2 multiplex rank gives a larger lower bound on rank than multilinear rank does.

Finally, it can be seen that $T$ is not folding generic. The determinantal schemes given by $M$ or $N$ are subschemes of $\mathbb{P}^{2} \times \mathbb{P}^{2}$, which has dimension 4. If $M$ and $N$ exhibited generic behavior, then the schemes defined by their $2 \times 2$ subdeterminants would have dimension 0 , that is, be finite sets of points. Using the computer program Maple, we were able to find a one-dimensional piece of the scheme defined by the $2 \times 2$ subdeterminants of $M$. Look on the affine patch of $\mathbb{P}^{2} \times \mathbb{P}^{2}$, where $w_{1}=x_{1}=1$. If we set:

$$
w_{2}=\left(-x_{3}^{2}-1\right)^{1 / 2}, \quad w_{3}=x_{3}, \quad x_{2}=\left(-x_{3}^{2}-1\right)^{1 / 2},
$$

we find that all the $2 \times 2$ subdeterminants of $M$ vanish. It is interesting that this locus is independent of $\lambda$ and $\mu$.

Acknowledgments. The authors would like to thank the referee for useful, insightful suggestions that helped improve the paper.

\section{REFERENCES}

1. J. Alexander and A. Hirschowitz, Polynomial interpolation in several variables, J. Alg. Geom. 4 (1995), 201-222.

2. E. Arbarello, M. Cornalba, P.A. Griffiths and J. Harris, Geometry of algebraic curves, Volume I, Grundl. Math. Wissen. 267, Springer-Verlag, New York, 1985.

3. Arnaud Beauville, Complex algebraic surfaces, Lond. Math. Soc. Lect. Note 68, Cambridge University Press, Cambridge, 1983.

4. W. Bruns and U. Vetter, Determinantal rings, Springer Lect. Notes Math. 1327, Springer Verlag, New York, 1988.

5. Enrico Carlini and Johannes Kleppe, Ranks derived from multilinear maps, J. Pure Appl. Alg. 215 (2011), 1999-2004.

6. Philippe G. Ciarlet, Mathematical elasticity, Volume 1: Three dimensional elasticity, North-Holland, Amsterdam, 1988.

7. Susan M. Cooper and Steven P. Diaz, The Gale transform and multi-graded determinantal schemes, J. Alg. 319 (2008), 3120-3127.

8. C. DeConcini, D. Eisenbud and C. Procesi, Young diagrams and determinantal varieties, Invent. Math. 56 (1980), 129-165.

9. __ Hodge algebras, Asterisque 91, Soc. Math. France (1982), 87 pages.

10. David Eisenbud, Commutative algebra with a view toward algebraic geometry, Grad. Texts Math. 150, Springer, New York, 1995.

11. David Eisenbud and Sorin Popescu, The projective geometry of the Gale transform, J. Alg. 230 (2000), 127-173. 
12. Joe Harris, Algebraic geometry, Grad. Texts Math. 133, Springer, New York, 1992.

13. Robin Hartshorne, Algebraic geometry, Grad. Texts Math. 52, Springer, New York, 1977.

14. F.L. Hitchcock, Multiple invariants and generalized rank of a p-way matrix or tensor, J. Math. Phys. 7 (1927), 39-79.

15. T.G. Kolda, Multilinear operators for higher-order decompositions, Tech. Report SAND 2006-2081, Sandia National Laboratories, Albuquerque, NM, 2006.

16. T.G. Kolda and B. Bader, Tensor decompositions and applications, SIAM Rev. 51 (2009), 455-500.

17. Alexei I. Kostrikin and Yu.I. Manin, Linear algebra and geometry, Gordon and Breach Science Publishers, New York, 1997.

18. J.M. Landsberg, Tensors: Geometry and applications, Grad. Stud. Math. 128, American Mathematical Society, Providence, 2012.

19. Jerrold E. Marsden and Thomas J.R. Hughes, Mathematical foundations of elasticity, Prentice-Hall, Englewood Cliffs, NJ, 1983.

20. V. De Silva and Lek-Heng Lim, Tensor rank and the ill-posedness of the best low-rank approximation problem, SIAM J. Matrix Anal. Appl. 30 (2008), 10841127.

Department of Mathematics, Syracuse University, Syracuse, NY 13244

Email address: spdiaz@syr.edu

Department of Mathematics, Syracuse University, Syracuse, NY 13244

Email address: alutobor@syr.edu 